\title{
Measurement of the average electromagnetic longitudinal shower profile at the Pierre Auger Observatory
}

\author{
Francisco Diogo $^{* a}$ for the Pierre Auger Collaboration ${ }^{b}$ \\ ${ }^{a}$ LIP, Av. Elias Garcia, $141^{\circ}$, Lisboa, Portugal \\ ${ }^{b}$ Observatorio Pierre Auger, Av. San Martín Norte 304, 5613 Malargüe, Argentina \\ E-mail: auger_spokespersons@fnal.gov \\ Full author list: http://www.auger.org/archive/authors_2015_06.html
}

In addition to the standard $X_{\max }$ and energy, the longitudinal profiles of extensive air showers contain some more interesting information. For energies above $10^{17.8} \mathrm{eV}$, we present the average profiles as a function of atmospheric depth measured for the first time at the Pierre Auger Observatory. The profile shapes for different energy ranges are all well reproduced by a GaisserHillas function within the range studied. A detailed analysis of the systematic uncertainties is performed using data and a full detector simulation, and the results are compared with predictions of hadronic interaction models for different primaries.

The 34th International Cosmic Ray Conference

30 July - 6 August, 2015

The Hague, The Netherlands

* Speaker. 


\section{Introduction}

Ultra High Energy Cosmic Rays (UHECRs) are the most energetic particles in the universe. The study of the cascades resulting from their interaction with the atmosphere nuclei gives us the only glimpse into hadronic interaction properties at these energies, more than an order of magnitude above those attained in man-made colliders.

While the atmospheric depth of shower maximum has long been used as a composition variable, the shape of the profile has remained largely untested.

In this paper we will present the first measurement of the average longitudinal profile in atmospheric depth (it has been previously been measured in age by HiRes/MIA and HiRes-II collaborations) for energies above $10^{17.8} \mathrm{eV}$. With the unprecedented statistics and experimental resolution of the Pierre Auger Observatory [1] at the highest energies, we can study the shower development in data, check the consistency of the reconstruction method, and also make a comparison with expectations from hadronic interaction models.

\section{Event reconstruction}

The Pierre Auger Observatory is a hybrid detector, consisting of a $3000 \mathrm{~km}^{2}$ Surface Detector array (SD) overlooked by the Fluorescence Detector (FD). The FD consists of four sites with 6 telescopes each and a fifth site with 3 telescopes (HEAT). The field of view of each telescope spans $30^{\circ}$ in azimuth and ranges from $1.5^{\circ}$ to $30^{\circ}$ in elevation (except for HEAT, where the elevation can be switched also to span $30^{\circ}$ to $60^{\circ}$ ). In this analysis we have not used information from HEAT. In addition to both detectors, there are also atmosphere monitoring tools which measure aerosol content, clouds and the temperature and density height profile.

The first step in the profile reconstruction is the determination of the shower geometry. Firstly, the SDP plane spanned by the pointing directions of pixels in the shower image is calculated. The shower axis within this plane is obtained using the timing information of each pixel, as well as the timing of the closest SD station (hybrid reconstruction). The opening angle in the perpendicular direction to the SDP $(\zeta)$ used for light integration is calculated by maximizing the signal to background ratio on an event-by-event basis. The light at the emission point is calculated taking into account the atmospheric characteristics measured autonomously.

Only the fluorescence component is proportional to the energy deposit in the shower volume considered, while Cherenkov light is dependent on the total number of particles above the emission energy threshold. Therefore, the complete profile has to be known for the calculation of the Cherenkov component. To extrapolate the directly observed profile to the earlier stages of development we use the Gaisser-Hillas (GH) function [2]

$$
f_{\mathrm{GH}}(X)=\left(\frac{\mathrm{d} E}{\mathrm{~d} X}\right)_{\max }\left(\frac{X-X_{0}}{X_{\max }-X_{0}}\right)^{\frac{X_{\max }-X_{0}}{\lambda}} \exp \left(\frac{X_{\max }-X}{\lambda}\right)
$$

which has four parameters: the maximum energy deposit, $(\mathrm{d} E / \mathrm{d} X)_{\max }$, the depth at which this maximum is reached, $X_{\max }$, and shape parameters $X_{0}$ and $\lambda$. Given the shower geometry w.r.t. the telescope, both the fluorescence and Cherenkov components can be simultaneously determined [3]. 


\section{Average Longitudinal Shower Profile}

The maximum energy deposit of the longitudinal profile, $(\mathrm{d} E / \mathrm{d} X)_{\max }$, is proportional to the energy of the primary particle and varies three orders of magnitude in the energy range studied in this work. $X_{\max }$ is characteristic of the primary mass, but also varies greatly within each primary group , mainly due to the stochastic nature of shower development and $X_{1}$, the depth at which the first interaction occurs.

To separate the information on these two parameters $\left((\mathrm{d} E / \mathrm{d} X)_{\max }\right.$ and $\left.X_{\max }\right)$ from the profile shape, which is the focus of this work, we first divide each measured shower profile by its fitted $(\mathrm{d} E / \mathrm{d} X)_{\max }$, rescaling all showers to have maxima at 1 . Then, we shift the $X$ axis, translating the atmospheric depth by $X_{\max }$, i.e., $X^{\prime} \equiv X-X_{\max }$, thus centering all profiles at zero. In previous analysis, done by the Hires/MIA [4] and HiRes-II Collaborations [5], the $X$ axis was scaled using shower age $\left(s=3 X /\left(X+2 X_{\max }\right)\right)$, and the resulting profiles were found to be compatible with a gaussian having RMS equal to $\sigma_{\text {age }}$. This width, however, is convolved with (and dominated by) the $X_{\max }$ value $^{1}$, hence in this work we choose to translate the profiles in atmospheric depth as it keeps the measured event-by-event shape unchanged.

We can write the Gaisser-Hillas function with these normalized variables, as a function of parameters $R$ and $L[6]$ :

$$
\begin{aligned}
\left(\frac{d E}{d X}\right)^{\prime} & =\exp \left(-\frac{1}{2}\left[\frac{X^{\prime}}{L}\right]^{2}\right) \prod_{n=3}^{\infty} \exp \left(-\frac{R^{n-2}}{n}\left[-\frac{X^{\prime}}{L}\right]^{n}\right) \\
& =\left(1+R \frac{X^{\prime}}{L}\right)^{R^{-2}} \exp \left(-\frac{X^{\prime}}{R L}\right)
\end{aligned}
$$

where $(\mathrm{d} E / \mathrm{d} X)^{\prime}=(\mathrm{d} E / \mathrm{d} X) /(\mathrm{d} E / \mathrm{d} X)_{\max }, R=\sqrt{\lambda /\left|X_{0}^{\prime}\right|}$ and $L=\sqrt{\left|X_{0}^{\prime}\right| \lambda}$ (note that $X_{0}^{\prime} \equiv X_{0}-$ $\left.X_{\max }\right)$. We choose these parameters because they are much less correlated than $X_{0}$ and $\lambda$ and have a more clear meaning: looking at equation (3.1) we see the GH function is a Gaussian with standard deviation $L$, multiplied by a term that distorts it, with the asymmetry governed by $R$ (i.e., if $R=0$, the function is a Gaussian). An equivalent parametrization as a function of the Full Width at Half Maximum, $f_{\mathrm{FWHM}}$, and asymmetry, $f$, has been reached independently [8].

The motivation for measuring the average profile shape is two-fold. First, it gives us a tool to control the quality of our reconstruction, and also to cross-check whether the assumption that showers are well described by a Gaisser-Hillas function is valid. Secondly, the profile shape carries information about the high energy hadronic interactions at the top of the atmosphere [7]. While the differences in $R$ and $L$ between proton and iron, or between different high energy hadronic models, are much smaller than those in $X_{\max }$, these variables give an independent measurement of the properties of the primary and its interacton in the atmosphere.

\footnotetext{
${ }^{1}$ We can write $s=1+\frac{2 X^{\prime}}{3 X_{\max }-X^{\prime}}$. If we say the RMS in age corresponds $X^{\prime}= \pm L$ and invert the equation, we get an approximation for $L$ as a function of $X_{\max }$ and $\sigma_{\mathrm{age}}: L_{\sigma}=\frac{3 X_{\max }}{\sigma_{\mathrm{age}}}\left(\sqrt{\left(1+\sigma_{\mathrm{age}}^{2}\right)}-1\right)$. This agrees with the true $\mathrm{L}$ within $0.5 \%$ at all energies. Re-writing this expression for $\sigma_{\text {age }}$ and making the derivatives w.r.t. $L$ and $X_{\max }$ we find that $\left(\frac{\partial \sigma_{\text {age }}}{\partial L} \Delta_{L}\right) /\left(\frac{\partial \sigma_{\text {age }}}{\partial X_{\max }} \Delta_{X_{\max }}\right)=\frac{X_{\max } \Delta_{L}}{L \Delta_{X_{\max }}} \approx 1 / 6$, so the majority of the separation in $\sigma_{\text {age }}$ comes from $X_{\max }$
} 

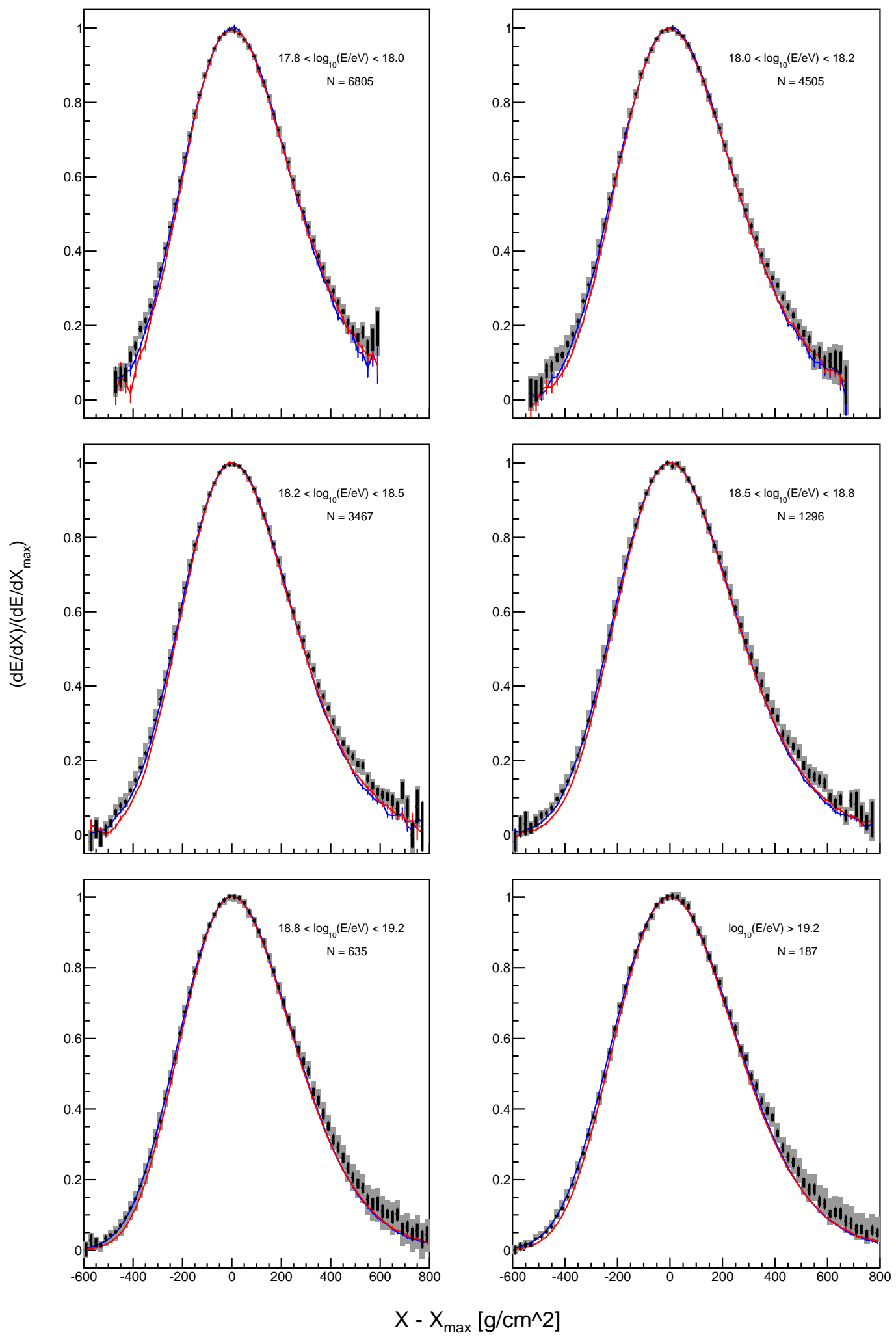

Figure 1: Average profiles for all the energy bins used in this work. The statistical error is shown as a black line and an estimation of the systematic uncertainty bin-by-bin as a gray area. Reconstructed profiles for $\mathrm{MC}$ are show in blue (proton) and re (iron). The high energy model used is QGSJETII03. 

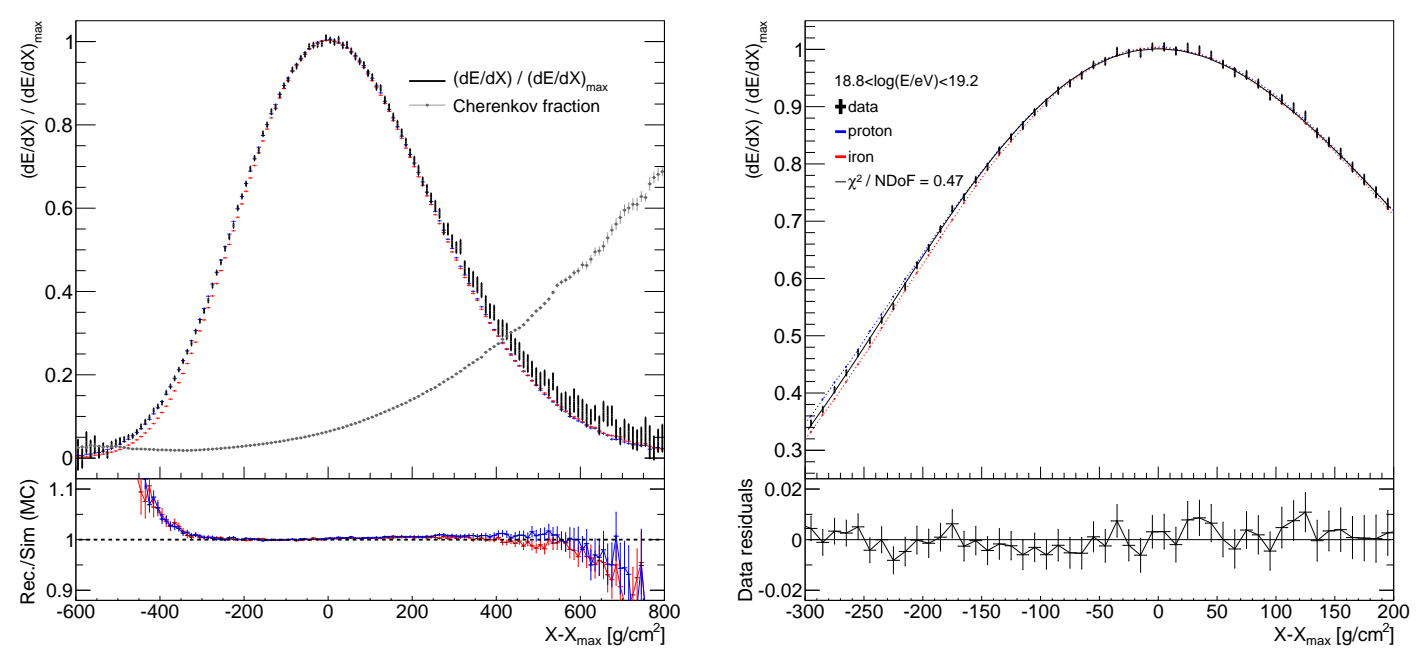

Figure 2: Left: Average profiles for energies between $10^{18.8}$ and $10^{19.2} \mathrm{eV}$. Data is shown in black, proton and iron reconstruction in blue and red respectively. The grey points represents the fraction of the total Cherenkov (direct and scattered) contribution in each bin. In the bottom plot is the ratio of reconstructed MC profiles over the generated ones for proton (blue) and iron (red). Right: The same profiles as on the left but only the fitting range is shown and the Gaisser-Hillas fit superimposed. The residuals of the fit to the data profile are shown in the bottom.

\section{Data selection and Monte Carlo validation}

The event selection used here is based on the most recent Auger Collaboration $X_{\max }$ analysis paper ([9]). The cuts used include requiring no clouds and an existing aerosol measurement, as well as a good hybrid geometry reconstruction. On the shower profile very strict cuts are made: at least $300 \mathrm{~g} / \mathrm{cm}^{2}$ must be observed, including the $X_{\max }$ depth, for which the expected resolution must be below $40 \mathrm{~g} / \mathrm{cm}^{2}$. A fiducial field of view is defined to guarantee the $\left\langle X_{\max }\right\rangle$ measurement is unbiased w.r.t. composition. Also, to minimize the amount of Cherenkov light, the minimum angle between a pixel pointing vector and the shower axis has to be larger than $20^{\circ}$. In this work, two additional cuts were used. One of the telescopes was excluded due to alignment problems, detected as significant time residuals found for showers crossing more than one telescope. Events in which more than $25 \%$ of the triggered pixels had large time residuals (greater than $3 \sigma$ ) from the geometry fit, were also not used. In the first cut we lose approximately $3 \%$ of the events (479), while in the second only 8 events were excluded.

In total 15782 events were selected, and we divide them in 6 energy bins. The shower profiles are constructed in $10 \mathrm{~g} / \mathrm{cm}^{2}$ bins in $X^{\prime}$, in which each energy deposit is accumulated with a weight corresponding to the inverse of its squared error. The profiles for all energies are shown in figure 1. Each average profile is then fitted with function (3.1).

This method was validated with a full detector simulation for energies between $10^{17}$ and $10^{20} \mathrm{eV}$ with proton and iron as primary particles. Comparing simulated and reconstructed average showers an excellent agreement was found for $X^{\prime}>-300 \mathrm{~g} / \mathrm{cm}^{2}$, but reconstruction deviated increasingly below it (see figure 2 (left) for the energy bin around $10^{19} \mathrm{eV}$ ). Above $X_{\max }$, showers 

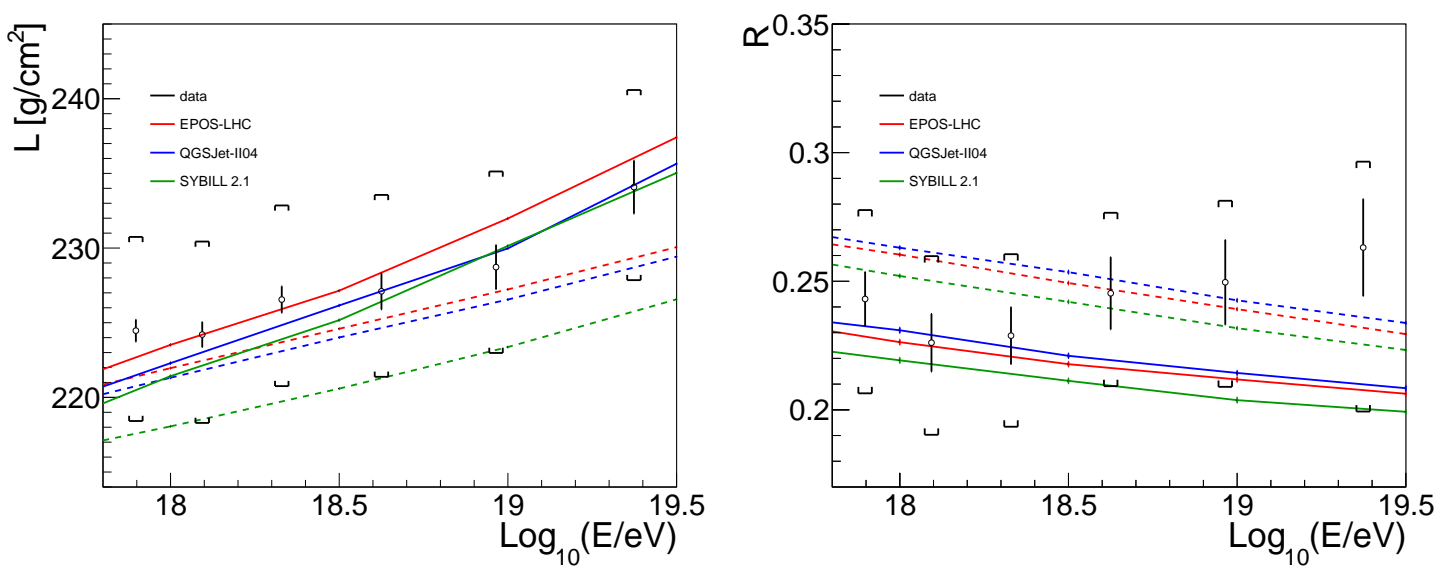

Figure 3: $L$ (left) and $R$ (right) as a function of energy. The data is shown in black, with the vertical line representing the statistical error and the brackets the systematic uncertainty. Hadronic interaction models are shown each with its color (see legend), with full lines being proton predictions and dashed lines iron ones.

lose most of the primary information (in [6], when trying to maximize proton-iron separation using only simulations, $100 \mathrm{~g} / \mathrm{cm}^{2}$ was chosen as upper fit limit) and the fluorescence light fraction falls rapidly (figure 2 (right)). Hence, the fit limits were chosen to be -300 to $+200 \mathrm{~g} / \mathrm{cm}^{2}$ since it allows having a statistical error smaller than the proton/iron separation at all energies while keeping the minimum fluorescence fraction around $80 \%$.

The reconstructed and simulated profiles are then fitted with equation (3.1) leaving all parameters unconstrained ${ }^{2}$. The fitted values for the shape parameters agree well between simulation and reconstruction for all energies above $10^{18} \mathrm{eV}$, with a larger difference for the first energy bin $\left(10^{17.8}\right.$ to $10^{18} \mathrm{eV}$ ). The average bias is corrected and half the proton-iron difference value is added to the reconstruction systematic uncertainty in Table 1.

\section{Systematic uncertainties}

The atmospheric conditions play a crucial role in the propagation of the light, so several systematic uncertainties related to it were studied: cloud effects, uncertainties of the overall aerosol content as well as its height dependence, the effect of excluding the outermost $1.5^{\circ}$ at the border of the camera from the fit and differences found when separating data by the seasons of the year. Since all these effects tend to change mostly either the beginning (clouds) or the end (aerosols) of the profile, they contribute strongly to the systematic uncertainty of the asymmetry $(R)$. We also considered the uncertainties in the determination of the fraction of measured light that corresponds to fluorescence, direct or scattered (Mie or Rayleigh) Cherenkov light and multiple scattering. This includes changing the fluorescence and Cherenkov yield value within its experimental uncertainty in the reconstruction, accounting or not for the multiple scattering corrections, and separating data according to the fluorescence fraction on the event. Among these, the largest effect found was that

\footnotetext{
${ }^{2}$ In addition to $R$ and $L$, also the normalization and the maximum are allowed to vary around 1 and 0 respectively, since in data we have smearing and small energy dependent bias. Values in data are always within the MC predictions, i.e., $<0.5 \%$ for normalization and less than $1 \mathrm{~g} / \mathrm{cm}^{2}$ for maximum
} 
showers with fluorescence fraction lower than the average (around 90\%) are approximately $4 \mathrm{~g} / \mathrm{cm}^{2}$ larger in width, $L$. The shape parameters for the individual shower, $X_{0}$ and $\lambda$ are constrained with a value measured in the data, so we changed these constraints by $1 \sigma$ in the reconstruction. We tested for systematic effects of the telescope alignment by studying the telescope-to-telescope differences of the reconstructed shape. Also, a dependence of $L$ and $R$ on the zenith angle or distance from $X_{\max }$ to the telescope was studied, but found to be relatively small in comparison to the previous ones. The uncertainty from the proton-iron difference in the reconstruction bias correction and the uncertainty of the energy scale of $14 \%$ [10] are also small.

\begin{tabular}{lcc}
\hline & $\boldsymbol{R}$ & $\boldsymbol{L}\left[\mathrm{g} / \mathrm{cm}^{2}\right]$ \\
\hline Atmosphere & 0.053 & 3.6 \\
Light components \& fit & 0.011 & 4.0 \\
Telescope & 0.023 & 3.2 \\
Geometry & 0.018 & 2.0 \\
Bias corr. \& Energy & 0.007 & 0.6 \\
Total & $\mathbf{0 . 0 6 3}$ & $\mathbf{6 . 3}$ \\
\hline Statistical & 0.019 & 1.8 \\
\hline
\end{tabular}

Table 1: Breakdown of systematic uncertainties for $R$ and $L$. Uncertatinties are energy dependent and asymmetric so that only the largest value is reported.

\section{Results}

The fit of data profiles to the Gaisser-Hillas function (3.1) is shown in figure 2 (right). The fitted function follows data points through the whole depth range used in this work, $[-300,+200]$ $\mathrm{g} / \mathrm{cm}^{2}$, with residuals always within the statistical uncertainty. The overall reduced $\chi^{2}$ is below 1.5 for all energies, showing that the analysis of the average shape of profiles in terms of the $R$ and $L$ parameters in equation (3.1) is an accurate description. The results of $L$ and $R$ as a function of energy are shown in figure 3. The width, $L$, in data agrees well with the predicted values for all models, and its energy evolution is consistent with a linear increase with $\log _{10}[E / \mathrm{eV}]$. The asymmetry, $R$, is compatible with models. In the data there is an increase with energy not predicted by them, although it is contained within the systematic uncertainty of the measurement.

It is also interesting to see the results in the $(R, L)$ plane for a fixed energy (Figure 4$)$. In these plots we can also represent all possible composition scenarios (as a combination of proton, $\mathrm{He}, \mathrm{N}$ and $\mathrm{Fe}$ ) for a given energy and make use of our knowledge about the correlation between $R$ and L. In Figure 4 (left), for a low energy bin, we can see the average value in data is in the area occupied by most models for a light composition, while at $10^{19} \mathrm{eV}$ (figure 4 (right)) it is within the predictions for heavier primaries. However, they are still fully compatible with all composition scenarios on $2 \sigma$ level, so the objective of future work is to decrease the systematic uncertainty and derive further constraints on predictions of hadronic models. 

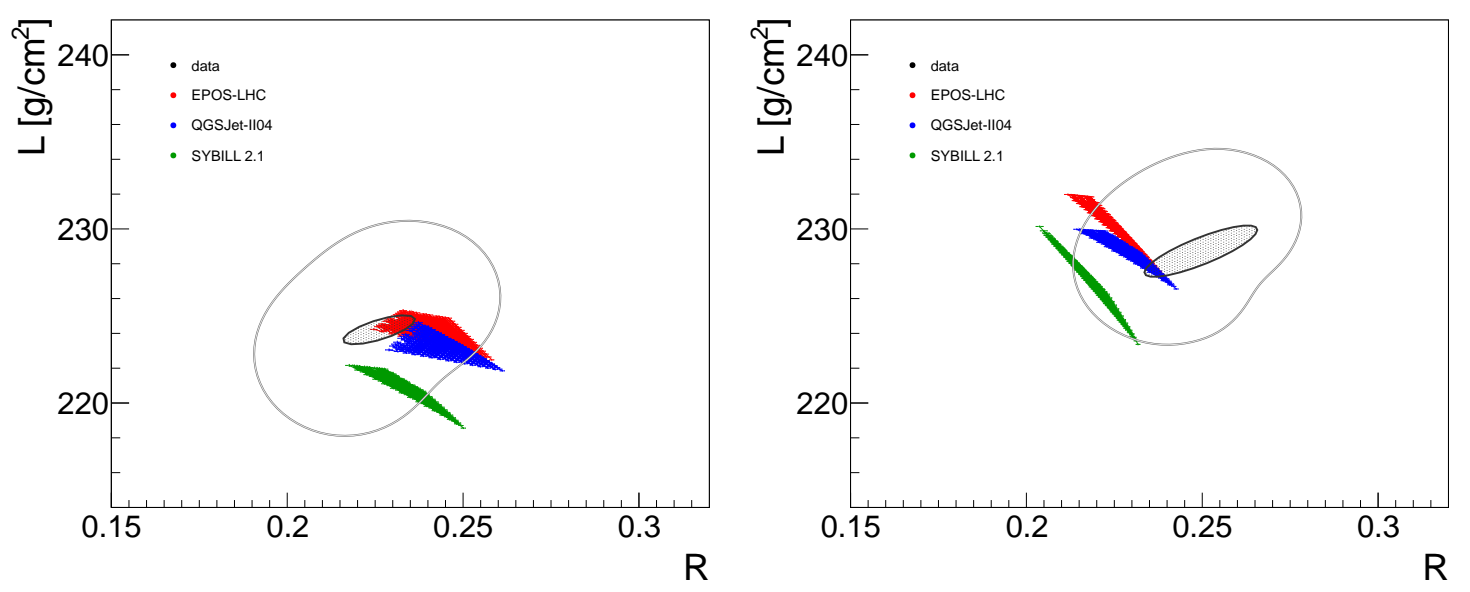

Figure 4: $L$ vs $R$ for the energy bin $10^{18}$ to $10^{18.2} \mathrm{eV}$ (left) and from $10^{18.8}$ to $10^{19.2} \mathrm{eV}$ (right). The inner dark grey ellipse shows the fitted value for data and its statistical error, and the outer light grey area the systematic uncertainty. For each hadronic model all combinations of proton, helium, nitrogen and iron were simulated and are represented by its respective colored area. Pure proton is, for each model, on the upper left side and the transition to iron goes gradually to the lower right one.

\section{Conclusion and Outlook}

In this work, the average shape of the longitudinal profile of the air showers in the Pierre Auger Observatory was measured. We first validated the method in a full detector simulation of proton and iron primaries, which showed that reconstructed and simulated profiles are in very good agreement for all energies above $10^{17.8} \mathrm{eV}$. We have shown that average profiles of the data are well described by a Gaisser-Hillas function through the entire fitting range chosen. We estimated the systematic uncertainties contributing to our measurement, and concluded that the atmospheric description and the Cherenkov contribution are the main factors that affect the asymmetry and the width of the profile, respectively. The two shape parameters, $R$ and $L$, resulting from this fit were compared with model predictions, being fully compatible with them.

\section{References}

[1] Pierre Auger Collaboration, A. Aab et al., The Pierre Auger Cosmic Ray Observatory, accepted for publication in Nucl. Instrum. Meth. A (2015), [arXiv: 1502.01323].

[2] T.K. Gaisser and A.M. Hillas, Proc. 15th ICRC, Plovdiv, Bulgaria 8 (1977) 353.

[3] M. Unger et al., Nucl. Instrum. Meth. A 588 (2008) 433, [arXiv: 0801.4309].

[4] The HiRes/MIA Collaboration, T. Abu-Zayyad et al., Astropart. Phys. 16 (2001) 1-11.

[5] G. Hughes for the High Resolution Fly's Eye Collaboration, Proc. 30th ICRC, Merida, Mexico 4 (2007) 405-408.

[6] S. Andringa, R. Conceição, and M. Pimenta, Astropart. Phys. 34 (2011) 360-367.

[7] R. Conceição et al., 24th European Cosmic Ray Symposium (2014) (to appear in JPCS).

[8] J.A.J. Matthews et al., J. Phys. G 37 (2010) 025202, [arXiv: 0909.4014].

[9] Pierre Auger Collaboration, A. Aab et al., Phys. Rev. D 90 (2014) 122005.

[10] V. Verzi for the Pierre Auger Collaboration, Proc. 33rd ICRC, Rio de Janeiro, Brazil (2013), [arXiv: 1307.5059]. 